\title{
Porphyria cutanea tarda in association with the human immunodeficiency virus infection
}

\author{
E.L.C. Ong, ${ }^{1}$ M.E. Ellis, ${ }^{1}$ D. McDowell, ${ }^{2}$ M. Gebril, ${ }^{2}$ C. Weinkove ${ }^{2}$ \\ and R. $\mathrm{Ead}^{3}$
} ${ }^{1}$ Regional Department of Infectious Diseases and Tropical Medicine, Monsall Hospital, Manchester
M10 8WR, ${ }^{2}$ Department of Chemical Pathology, Hope Hospital, Eccles Old Road, Salford M6 8HD
and ${ }^{3}$ Department of Dermatology, Monsall Hospital, Manchester M10 8WR, UK.

\begin{abstract}
Summary: A 42 year old heterosexual male with symptomatic human immunodeficiency virus infection presented with a 2 -week history of tense blistering skin lesions following azidothymidine therapy. Urinary porphyrin excretion confirmed the diagnosis of porphyria cutanea tarda. The blisters resolved following the withdrawal of the drug but recurred when rechallenged. Three other cases of porphyria cutanea tarda, not associated with azidothymidine, who subsequently developed acquired immunodeficiency syndrome have recently been described. If azidothymidine is not the precipitating agent, it is possible that human immunodeficiency virus itself can impair porphyrin metabolism, leading to the clinical and biochemical features of porphyria cutanea tarda.
\end{abstract}

\section{Introduction}

The incidence of acquired immunodeficiency syndrome (AIDS) is yet unclear, but as more groups of individuals become susceptible, patterns of accompanying disease states have increasingly been recognized. We report a case of porphyria cutanea tarda (PCT) associated with the human immunodeficiency virus (HIV).

\section{Case report}

A 42 year old heterosexual male with symptomatic HIV infection presented in July 1987 with a 2-week history of tense blistering skin lesions on the dorsal aspects of his hands and fingers.

He was diagnosed antibody positive (ELISA and Western blot) to HIV in September 1986 when his main problems were intermittent fever, lethargy, oral candidiasis and chronic nail dystrophy affecting finger-nails of both hands. He gave a history of heterosexual contact with a prostitute in Central Africa in 1982.

His drug history included 8 weeks of griseofulvin for tinea infection, and fluconazole for oral candidiasis in September 1986, 3 weeks of co-trimoxazole (Septrin) for suspected but unproven Pneumocystis

Correspondence: $\quad$ E.L.C. $\quad$ Ong, $\quad$ M.Sc., $\quad$ M.R.C.P.,

D.T.M.\& H.

Accepted: 7 July 1988 carinii pneumonia in January 1987. Although he consumed 4 pints of beer daily in the past, he had been abstinent for over 2 years. Azidothymidine (AZT) had been introduced for 2 weeks prior to the development of the blisters.

On admission the haemoglobin was $9.1 \mathrm{~g} / \mathrm{dl}$, white cell count $2.6 \times 10^{9} / 1$ with $64 \%$ neutrophils, $30 \%$ lymphocytes, $2 \%$ monocytes, $4 \%$ eosinophils. Electrolytes, urea and creatinine were normal. Liver function tests showed alkaline phosphatase $126 \mathrm{IU} / 1$ (normal 35-105), alanine transaminase $40 \mathrm{IU} / 1$ (normal 0-35) and gamma glutamyltransferase $203 \mathrm{IU} / 1$ (normal $0-30$ ). Serum iron $10 \mu \mathrm{mol} / 1$ (1226) and total iron binding capacity $45 \mu \mathrm{mol} / 1$ (4570) saturation $22 \%$. A random urine sample was found to contain uroporphyrin $7090 \mathrm{nmol} / 1$ (normal $<38$ ) and 7-carboxyporphyrin $4760 \mathrm{nmol} / 1$ (normal $<14$ ), (see Figure 1). Abdominal ultrasound showed markedly fatty liver with normal biliary tree and pancreas. Liver biopsy showed the portal tracts to be within normal limits. In the parenchyma, there was moderate steatosis, focal neutrophilic infiltrate, frequent Mallory bodies, pericellular and perivenular fibrosis, and parenchymal and Kupffer cell siderosis. The-overall picture was that of alcoholic type hepatitis with the urinary porphyrin excretion pattern consistent with PCT.

AZT was stopped on presentation and the blisters resolved spontaneously over the course of one week. He has since been rechallenged with

(C) The Fellowship of Postgraduate Medicine, 1988 


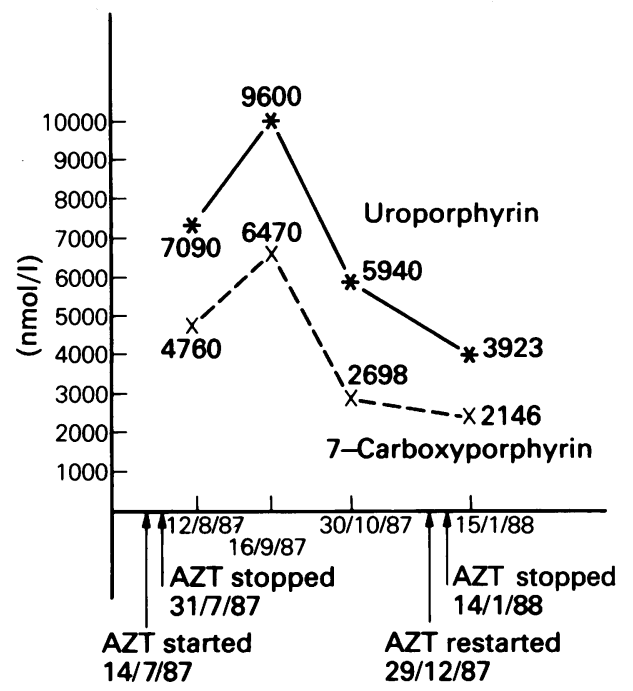

Figure 1 Urinary porphyrins. *-*, uroporphyrin; $x--x, 7$-carboxyporphyrin.

AZT and the blisters recurred within 2 weeks (see Figure 1).

\section{Discussion}

Porphyria cutanea tarda (PCT) is a relatively rare metabolic disorder. It is however the most common of the porphyric diseases in man. ${ }^{1}$ The defect in this disorder is a decreased activity of hepatic uroporphyrinogen decarboxylase in hepatic cells and erythrocytes in inherited form and hepatic cells in sporadic form. ${ }^{2}$ Cutaneous photosensitivity resulting in the development of subepidermal blisters is a major feature of PCT. Other cutaneous features are increased skin fragility which often precedes the development of blisters, milia formation, scarring, facial hirsutes and scleroderma-like

\section{References}

1. Kappas, A., Sassa, S. \& Anderson, K.E. The porphyrias. In: Stansbury, J.B., Wyngaarden, J.B., Frederickson, D.S., Goldstein, J.L., \& Brown, M.S. (eds) The Metabolic Basis of Inherited Disease, 5th edition. McGraw-Hill, New York, 1982, pp 1301.

2. Elder, G.H. Porphyria metabolism in porphyria cutanea tarda. Semin Haematol 1977, 14: 227.

3. Donahue, R.E., Johnson, M.M., Zon, L.I., Clark, S.C. \& Groopman, J.E. Suppression of in vitro haematopoiesis following human immunodeficiency virus factors. Nature 1987, 326: 200-203. changes. Opportunistic infections or haematological disorders are not associated with PCT but the disease is known to be associated with hepatocellular damage and there is a risk of hepatocellular carcinoma.

The anaemia present in this patient, which may be due to the HIV infection ${ }^{3}$ and/or the AZT therapy indicates that he has a diminished haem pool and, therefore, a reduction of the normal suppression of the rate limiting enzyme, aminolaevulinic acid (ALA) synthase. This leads to an increased availability of substrate exaggerating any defect in the enzymes further along the synthetic pathway, as these enzymes become rate limiting. Thus any slight deficiency of uroporphyrinogen decarboxylase activity, due to genetic causes, liver dysfunction or inhibition by drugs would produce the biochemical pattern of PCT.

In the absence of a family history of PCT, we feel that the patient's condition was acquired. Alcohol was an unlikely cause in this man since he had abstained for over two years. AZT was the sole medication prescribed prior to his symptoms, and his cutaneous signs resolved when this drug was withdrawn. On rechallenge with AZT, the dermatological features recurred. However, despite clinical improvement while off medication, his urinary porphyrin excretion remained grossly abnormal. We cannot exclude the possibility that the recurrence of the blisters may have been caused by further exposure to the ultraviolet light.

Three cases of PCT who subsequently also developed AIDS have recently been reported. ${ }^{4}$ AZT is not, however, a recognized cause of PCT. If AZT is not the precipitating agent, it is possible that HIV itself can impair porphyrin metabolism, leading to the clinical and biochemical features of PCT.

\section{Acknowledgement}

We thank Pfizer Ltd for the supply of fluconazole.

4. Wissel, P.S., Sordillo, P., Anderson, K.E., Sassa, S., Savillo, R.L. \& Kappas, A. Porphyria cutanea tarda associated with the acquired immune deficiency syndrome. Am J Hematol 1987, 25: 107-113. 